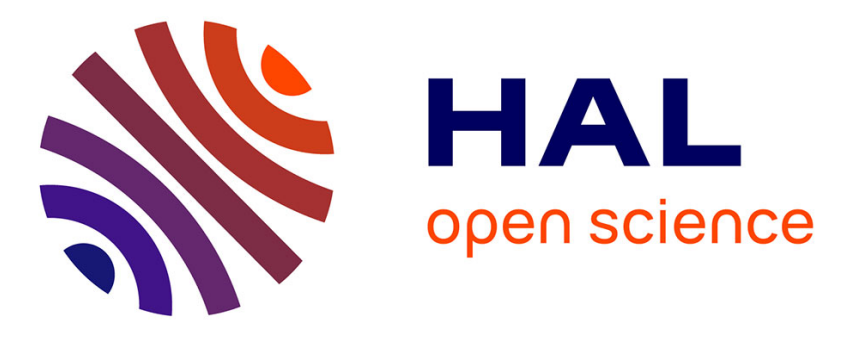

\title{
Scaffolding skeletons using spherical Voronoi diagrams
}

\author{
Alvaro Javier Fuentes Suárez, Evelyne Hubert
}

\section{To cite this version:}

Alvaro Javier Fuentes Suárez, Evelyne Hubert. Scaffolding skeletons using spherical Voronoi diagrams. LAGOS'17 - IX Latin and American Algorithms, Graphs and Optimization, Frédérique Bassino; Flavia Bonomo; Lionel Pournin; Mario Valencia-Pabon; Juan Carlos Vera Lizcano, Sep 2017, Marseille, France. pp.45-50, 10.1016/j.endm.2017.10.009 . hal-01509919v3

\section{HAL Id: hal-01509919 https://hal.inria.fr/hal-01509919v3}

Submitted on 6 Jun 2017

HAL is a multi-disciplinary open access archive for the deposit and dissemination of scientific research documents, whether they are published or not. The documents may come from teaching and research institutions in France or abroad, or from public or private research centers.
L'archive ouverte pluridisciplinaire HAL, est destinée au dépôt et à la diffusion de documents scientifiques de niveau recherche, publiés ou non, émanant des établissements d'enseignement et de recherche français ou étrangers, des laboratoires publics ou privés. 


\title{
Scaffolding skeletons using spherical Voronoi diagrams
}

\author{
A.J. Fuentes Suárez ${ }^{1,2}$ E. Hubert \\ Inria Sophia Antipolis
}

\begin{abstract}
Given a skeleton made of line segments we describe how to obtain a coarse mesh (or scaffold) of a surface surrounding it. We emphasize in this abstract the key result that allows us to complete the approach in [3] that could not treat skeletons with cycles.
\end{abstract}

Keywords: mesh generation, skeletal models, 3D modeling, Voronoi diagrams

\section{Introduction}

Skeletons are used in 3D graphics for modeling and animating articulated shapes. The user can design a complex shape by sketching a simple and intuitive geometric object that is the input to a surface generating algorithm. In the context of this paper a skeleton is a set of spatial line segments that do not intersect except at the endpoints, then called joints. It is used to mathematically define a surface around the skeleton: by convolution, by offset, or other kind of distance surface [13]. Once a surface is properly defined it

1 This project has received funding from the European Union's Horizon 2020 research and innovation programme under the Marie Skłodowska-Curie grant agreement No 675789 .

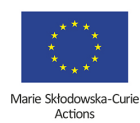

2 Email: alvaro.fuentes-suarez@inria.fr 
must be translated into an alternative representation either for visualization (mesh) or for local editing (NURBs, splines, etc). The issue is that usually the new representation is not related to the original skeleton and so the user loses control over the model. To overcome this a skeleton-based mesh generation method is needed. The idea developed here is to construct a "coarse" mesh around the skeleton, as regular as possible, and resembling the structure of the skeleton. This mesh is then used to generate the representation of the mathematical surface. Following the terminology of [11] we call this process scaffolding and the corresponding coarse mesh a scaffold.

One of the proposed ideas for the mesh reconstruction was the method of B-meshes [9], it creates a rectangular cage around each piece of the skeleton that are stitched together to make a closed mesh. The main drawback of this method is that the stitching process could lead to irregularities at the joints. Recently, Bærentzen et al. [3] proposed a method called SQM (Skeleton to Quad-dominant polygonal Mesh) going in the opposite direction: they first define the mesh points around the joints and then recreate a quad based "tubular" polyhedral surface around each line segment. SQM consists of two steps:

(i) Partition the unit sphere centered at the joints into cells, one for each incident line segment; each cell is defined by an ordered set of points on the sphere.

(ii) Create sleeves, i.e. link the vertices of the cells at the extremities of each line segment.

For the creation of the sleeves the extremal cells must be compatible, that is they must have the same number of vertices. The partition of the sphere in Bærentzen et al. [3] can be recognized to be a Voronoi diagram on the sphere. In order to match the cells they propose an algorithm for inserting additional vertices on the boundary of the cells in such a way that all the sleeves can be simultaneously created. Yet this algorithm does not work in the presence of cycles. Furthermore there is no analysis on the optimality of SQM with respect to the number of links.

The contribution we emphasize in this abstract is that we prove the process outlined above is feasible even in the presence of cycles. We present a method which uses Integer Linear Programing and computes an optimal solution minimizing the number of vertices for the partition of the sphere at the joints. Feasibility is proven thanks to a numerical characterization by Rivin [12] of graphs combinatorially equivalent to inscribed polyhedrons. Our minimization criterion is what gives the "coarsest" mesh, among those based 
on Voronoi partitions of the spheres at the joints.

It is worth mentioning that another partition of the sphere is introduced in [11], a partition into quadrangles, which therefore makes the matching of cells trivial, the caveat being that the convexity of the cells is not guaranteed.

\section{Formalization of the geometrical problem}

In this paper a skeleton is a finite set $\mathcal{S}$ of spatial line segments satisfying the following property: any two line segments intersect at most at one of their endpoints. A skeleton $\mathcal{S}$ defines naturally a graph $G_{\mathcal{S}}=\left(V_{\mathcal{S}}, E_{\mathcal{S}}\right.$ ) (following the notation in [6]), by identifying the set of vertices $V_{\mathcal{S}}$ with the set of all endpoints in $\mathcal{S}$, and the set of edges $E_{\mathcal{S}}$ with the set $\mathcal{S}$ itself. The graph $G_{\mathcal{S}}$ will be called the skeleton graph. An edge $e \in E_{\mathcal{S}}$ connecting two nodes $a, b \in V_{\mathcal{S}}$ can be alternatively represented as $a b$. If $e=a b$ we say that $e$ is incident to $a$ (or $b$ ) and will be denoted $e \multimap a$ (or $e \multimap b$ ). $s_{e}$ denotes the line segment in $\mathcal{S}$ associated to $e$. The unit sphere centered at a node $v \in V_{\mathcal{S}}$ is denoted $\mathbb{S}_{v}$, and $\mathcal{A}_{v}=\left\{s_{e} \cap \mathbb{S}_{v} \mid e \in E_{\mathcal{S}}, e \multimap v\right\}$ is the set of the intersection points of the line segments incident to $v$ with the unit sphere centered at $v$.

The Voronoi diagram [2] of $\mathcal{A}_{v}$ on the sphere, denoted $\operatorname{Vor}\left(\mathcal{A}_{v}\right)$, partitions the sphere $\mathbb{S}_{v}$ into regions $\left\{R_{e}^{v}\right\}_{e-o v}$, where $\left(\mathbb{S}_{v} \cap s_{e}\right) \in R_{e}^{v}$, that are delimited by arcs of great circles $[1,10]$. A region $R_{e}^{v}$ is thus well defined by an ordered set of points on $\mathbb{S}_{v}$ that we call the cell $C_{e}^{v}$. This set of points consists of the end points of the arcs delimiting $R_{e}^{v}$ and at least one additional point per arc to remove ambiguity. The number of points chosen on a $\operatorname{arc}$ of $C_{e}^{v}$ will be called the number of subdivisions for that arc.

A scaffold $\mathcal{K}_{\mathcal{S}}$ is defined as a pair $\left(P_{\mathcal{S}}, \Phi_{\mathcal{S}}\right)$, satisfying

(1) $P_{\mathcal{S}}=\left\{\mathcal{C}_{v} \mid v \in V_{\mathcal{S}}\right\}$ where each $\mathcal{C}_{v}=\left\{C_{e}^{v} \mid v \in V_{\mathcal{S}}, e \in E_{\mathcal{S}}, e \multimap v\right\}$ is a family of cells representing a partition of $\mathbb{S}_{v}$ according to $\operatorname{Vor}\left(\mathcal{A}_{v}\right)$.

(2) $\Phi_{\mathcal{S}}=\left\{\phi_{e} \mid e \in E_{\mathcal{S}}\right\}$ is a family of bijections $\phi_{e}$ between $C_{e}^{a}$ and $C_{e}^{b}$ for $e=a b$.

For $e \multimap v$ and $C_{e}^{v}=\left(p_{1}, p_{2}, \ldots, p_{n}\right)$ we say that $p_{i}$ is linked with $\phi_{e}\left(p_{i}\right)$. The term scaffold is "visually" justified by the following: if we try to visualize $\mathcal{K}_{\mathcal{S}}$ by drawing a line segment between any pair of linked elements of $P_{\mathcal{S}}$ we can see a structure that resembles a set of "scaffolds" around the skeleton $\mathcal{S}$. Our goal is to prove that the Voronoi cells can always be subdivided so as to build a scaffold. For that we need the cells $C_{e}^{a}$ and $C_{e}^{b}(e=a b)$ to have identical number of points. The point insertion strategy described in [3] does not work in the presence of cycles in the skeleton. 


\section{$3 \quad$ Feasibility and optimality}

We model the problem of matching the cells as an Integer Linear Program (IP) [5]. We shall prove first that the IP is feasible. Thus we can provide an optimal solution using the Branch-and-Bound algorithm [5, page 278].

Let $\mathcal{A}$ be a set of points on a 2 dimensional sphere and $\operatorname{Vor}(\mathcal{A})$ its 2 dimensional spherical Voronoi diagram. The Delaunay triangulation $\operatorname{Del}(\mathcal{A})$ is the dual of $\operatorname{Vor}(\mathcal{A})[2]$. $\operatorname{Vor}(\mathcal{A})$ is also dual to the spatial convex hull of $\mathcal{A}$ in the surrounding 3 dimensional space $[4,8]$. This means that the Delaunay triangulation is combinatorially equivalent to the convex hull.

For each $v \in V_{\mathcal{S}}$ let $E_{v}$ be the set of edges of $\operatorname{Del}\left(\mathcal{A}_{v}\right)$. Each edge in $E_{v}$ represents a common boundary between two regions in $\operatorname{Vor}\left(\mathcal{A}_{v}\right)$. For $f \in$ $E_{v}$ we define a positive integer $x_{f}^{v}$ called weight representing the number of subdivisions to be done to the corresponding arc. The number of elements in a cell $C_{e}^{v}\left(v \in V_{\mathcal{S}}, e \in E_{\mathcal{S}}\right.$ and $\left.e \multimap v\right)$ is given by

$$
\left|C_{e}^{v}\right|=\sum_{\substack{f \in E_{v} \\ f-0\left(\mathbb{S}_{v} \cap s_{e}\right)}} x_{f}^{v}
$$

For $e=a b \in E_{\mathcal{S}}$ the bijection $\phi_{e} \in \Phi_{\mathcal{S}}$ tells us that the cells $C_{e}^{a}$ and $C_{e}^{b}$ in $\mathcal{K}_{\mathcal{S}}$ must have the same number of elements. On the other hand the total number of links in $\mathcal{K}_{\mathcal{S}}$ is given by the sum of all the weights, thus our IP is formalized as follows.

$$
\begin{aligned}
& \text { Minimize: } \sum_{\substack{h \in E_{v} \\
v \in V_{\mathcal{S}}}} x_{h}^{v} \text { Subject to: } \sum_{\substack{f \in E_{a} \\
f-0\left(\mathbb{S}_{a} \cap s_{e}\right)}} x_{f}^{a}=\sum_{\substack{g \in E_{b} \\
g-0\left(\mathbb{S}_{b} \cap s_{e}\right)}} x_{g}^{b}, \quad \forall \phi_{e} \in \Phi_{\mathcal{S}} \\
& x_{h}^{v} \in \mathbb{Z}, x_{h}^{v} \geq 1, \quad \forall h \in E_{v}, v \in V_{\mathcal{S}}
\end{aligned}
$$

If this IP is feasible then it will have at least one optimal solution. The feasibility of the system (1) is the main result presented in this abstract, which we state in the following theorem.

Theorem 3.1 The set of linear equations (1) has a solution with all the entries $x_{h}^{v}$ in the set of positive integers.

The idea of the proof is to show that for $v \in V_{\mathcal{S}}$, the local linear system

$$
\sum_{\substack{f \in E_{v} \\ f-0\left(\mathbb{S}_{a} \cap s_{e}\right)}} x_{f}^{v}=\lambda_{v} \quad \forall e \multimap v, e=a b \in E_{\mathcal{S}}
$$


has a solution in the positive integers with $\lambda_{v}$ also a positive integer. The values $\tilde{x}_{f}^{v}$ along with $\tilde{\lambda}_{v}$ satisfying (2) will be called local solution. Indeed assuming there are local solutions $\left(\tilde{x}_{f}^{v}, \tilde{\lambda}_{v}\right)$ for all $v \in V_{\mathcal{S}}$ then there is a global solution $\hat{x}_{f}$ given by $\hat{x}_{f}=\hat{x}_{f}^{v}=\left(\lambda / \tilde{\lambda}_{v}\right) \cdot \tilde{x}_{f}^{v}$ where $\lambda=\prod_{u \in V_{\mathcal{S}}} \tilde{\lambda}_{u}$.

The existence of a real positive solution for the local system (2) comes from the fact that $\operatorname{Del}\left(\mathcal{A}_{v}\right)$ is combinatorially equivalent to the convex hull of $\mathcal{A}_{v}$ which is an inscribable polyhedron, and the following numerical characterization due to Rivin [12] (statement extracted from [7]).

Proposition 3.2 If a graph is of inscribable type then weights $w$ can be assigned to its edges such that:

(i) For each edge e, $0<w(e)<1 / 2$.

(ii) For each vertex $v$, the total weight of all edges incident to $v$ is equal to 1 .

One then argues that if there is a real positive solution then there must be also an integer positive solution.

\section{Application and examples}

The method outlined in this paper has been implemented and used successfully for scaffolding skeletons. It has been tested for a wide variety of models including closed skeletons. It is integrated in a project aiming at the visualization of convolution surfaces. Some results are shown in Figure 1.
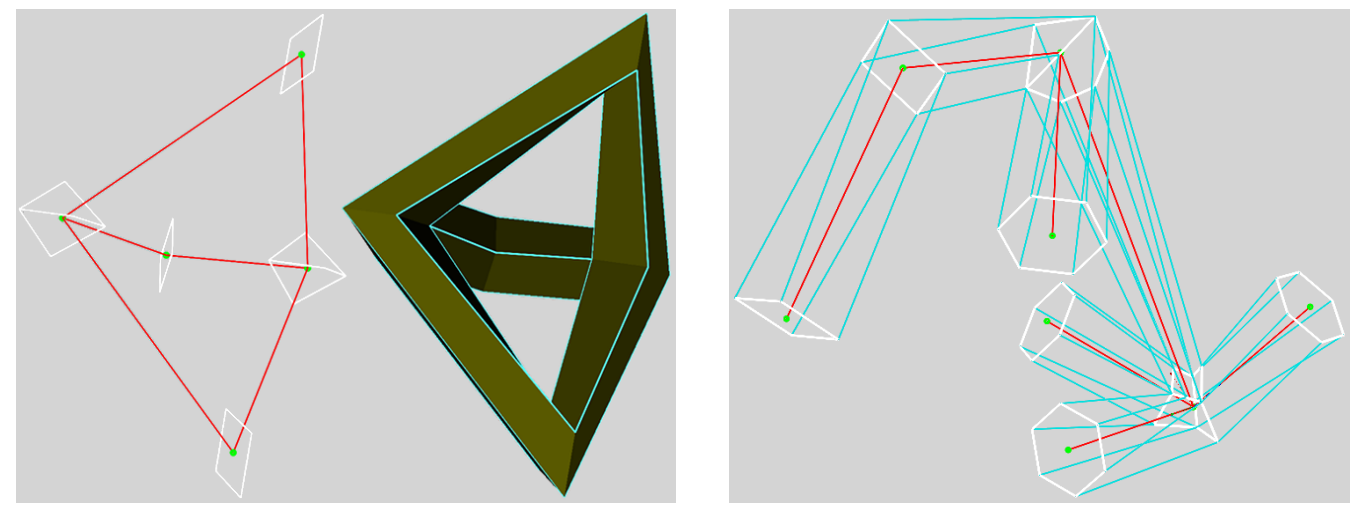

Fig. 1. Example skeletons and scaffolds (skeletons in red, endpoints in green, Voronoi cells in white, links in cyan, scaffold-based mesh in dark green). 


\section{References}

[1] Augenbaum, J. M. and C. S. Peskin, On the construction of the Voronoi mesh on a sphere, Journal of Computational Physics 59 (1985), pp. 177-192.

[2] Aurenhammer, F., Voronoi Diagrams A Survey of a Fundamental Data Structure, ACM Computing Surveys 23 (1991).

[3] Bærentzen, J. A., M. K. Misztal and K. Wełnicka, Converting skeletal structures to quad dominant meshes, Computers and Graphics 36 (2012).

[4] Brown, K. Q., "Geometric Transforms for Fast Geometric Algorithms," Phd thesis, Carnegie-Mellon University (1979).

[5] Chen, D.-S., R. G. Batson and Y. Dang, "Applied integer programming: modeling and solution," John Wiley \& Sons, 2011.

[6] Diestel, R., "Graph Theory," 1997, 3rd edition.

[7] Dillencourt, M. B. and W. D. Smith, Graph-theoretical conditions for inscribability and Delaunay realizability, Discrete Mathematics 161 (1996), pp. 63-77.

[8] Grima, C. I. and A. Márquez, "Computational Geometry on Surfaces," Springer Netherlands, 2001.

[9] Ji, Z., L. Liu and Y. Wang, B-Mesh: A modeling system for base meshes of $3 D$ articulated shapes, Computer Graphics Forum 29 (2010).

[10] Na, H.-S., C.-N. Lee and O. Cheong, Voronoi diagrams on the sphere, Computational Geometry 23 (2002).

[11] Panotopoulou, A., K. Welker, E. Ross, E. Hubert and G. Morin, Scaffolding a Skeleton .

URL https://hal.inria.fr/hal-01532765

[12] Rivin, I., A Characterization of Ideal Polyhedra in Hyperbolic 3-Space, Annals of Mathematics 143 (1996).

[13] Zanni, C., "Skeleton-based Implicit Modeling \& Applications," Phd thesis, Université de Grenoble (2013). 